\title{
Dynamics of phononic dissipation at the atomic scale: Dependence on internal degrees of freedom
}

\author{
H. Sevinçli, ${ }^{1}$ S. Mukhopadhyay, ${ }^{1, *}$ R. T. Senger,${ }^{1,2}$ and S. Ciraci ${ }^{1,2, \dagger}$ \\ ${ }^{1}$ Department of Physics, Bilkent University, 06800 Ankara, Turkey \\ ${ }^{2}$ UNAM-Material Science and Nanotechnology Institute, Bilkent University, 06800 Ankara, Turkey \\ (Received 1 March 2007; revised manuscript received 3 October 2007; published 21 November 2007)
}

\begin{abstract}
Dynamics of dissipation local vibrations to the surrounding substrate is a key issue in friction between sliding surfaces as well as in boundary lubrication. We consider a model system consisting of an excited nano-particle which is weakly coupled with a substrate. Using three different methods, we solve the dynamics of energy dissipation for different types of coupling between the nanoparticle and the substrate, where different types of dimensionality and phonon densities of states were also considered for the substrate. In this paper, we present a microscopic analysis of transient properties of energy dissipation via phonon discharge toward the substrate. Finally, important conclusions of our theoretical analysis are verified by a realistic study, where the phonon modes and interaction parameters involved in the energy dissipation from an excited benzene molecule to the graphene are calculated by using first-principles methods. The methods used are applicable also to dissipative processes in the contexts of infrared Raman spectroscopy and atomic force microscopy of molecules on surfaces.
\end{abstract}

DOI: 10.1103/PhysRevB.76.205430

PACS number(s): 68.35.Af, 63.22.+m

\section{INTRODUCTION}

Friction between two surfaces in relative motion involves many interesting and complex phenomena induced by the long- and short-range forces, such as adhesion, wetting, atom exchange, bond breaking and bond formation, and elastic and plastic deformation. ${ }^{1-14}$ In general, nonequilibrium phonons are generated in the expense of damped mechanical energy. ${ }^{15-20}$ Dissipation of this excess energy is one of the important issues in dry-sliding friction and lubrication. ${ }^{10,21-23}$ Normally, the dissipation of mechanical energy is resulted in heating of parts in relative motion. Sometimes, it gives rise to wear and failure due to overheating. In general, significant amounts of resources (energy and material) are lost in the course of friction. One of the prime goals of tribology is to minimize energy dissipation through lubrication. Recently, several works have attempted to develop surfaces with superlow friction coefficients. ${ }^{24}$

In the past, the energy dissipation during sliding has been usually investigated in the macroscopic scale by using simple Tomlinson's model. ${ }^{3}$ Hence, the dissipated energy and friction force have been revealed indirectly from stick-slip motion. The objective of the present work is to develop a microscopic (or atomic scale) understanding of phononic energy dissipation during sliding friction, especially to shed some light on the dynamics of discharge of excited phonons on a nanoparticle (representing a lubricant molecule or an asperity) into the substrate. This problem has many aspects and the solution will depend on a variety of physical parameters which can be grouped into major categories, such as internal degrees of freedom of the nanoparticle, density of substrate phonon modes, the type and strength of coupling between the nanoparticle and the substrate, and finally the initial temperatures. One way of studying this problem could be carrying out state-of-the-art molecular dynamics simulations which yields sample specific results only. However, to explore the general features of the phononic dissipation, we propose an Hamiltonian treatment of the problem. Since the number of physical ingredients determining the dynamics is considerably large, our strategy will be to focus on them separately to reveal their role in energy dissipation.

In this work, we present our analysis concerning the dependence of phononic dissipation on internal degrees of freedom of the nanoparticle and the substrate by using two types of coupling between the finite and extended systems. We consider three different theoretical methods; namely, the equation of motion (EoM) technique which involves Laplace transforms for the solution of the coupled differential equations for phonon operators, the Fano-Anderson (FA) method which is useful for diagonalizing quadratic Hamiltonians, and Green's function (GF) method by which we can incorporate the effect of multimodes into the study. The first two methods have limited applicability for specific cases only and will be presented for completeness. The GF method, being the most general method, will be extensively discussed. We also note that the GF method allows solutions beyond linear response where the EoM and FA methods yield solutions within the linear response regime.

The organization of the paper is as follows. In Sec. II, we describe the physical model. The theoretical methods to be used and their limitations are presented in Sec. III. The applications of theoretical methods to different types of coupling and substrates having different densities of states will be presented and discussed in Sec. IV. These are mainly the dependence of the decay rate on the coupling constant, the interaction-specific dependence of decay rate on the nanoparticle mode frequencies, and the effect of neighboring modes on the decay rate of each other. Finally, we present a specific and realistic example, where the dissipation of vibrational modes of benzene molecule $\left(\mathrm{C}_{6} \mathrm{H}_{6}\right)$ to a graphene substrate is analyzed by using density functional theory (DFT) calculations. We summarize our conclusions in Sec. V. 


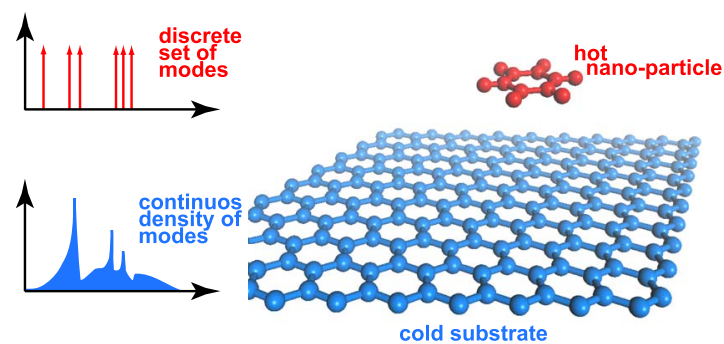

FIG. 1. (Color online) A nanoparticle with discrete density of phonon modes is coupled to a substrate having continuous density of modes.

\section{MODEL}

We first consider a nanoparticle representing a lubricant molecule or an asperity, which is weakly coupled to a substrate (Fig. 1). The vibrational modes of the nanoparticle are excited initially and the excess phonons discharge to the bulk. The total Hamiltonian of the system can be written as

$$
H=H_{M}+H_{S}+H_{M S}
$$

where $H_{M}$ and $H_{S}$ are the free phononic Hamiltonians of the nanoparticle (or molecule) and the substrate (or bath), respectively; the interaction between them is represented by $H_{M S}$. We also define $H=H_{0}+H_{M S}$. We assume that the harmonic approximation is good enough for $H_{M}$ and $H_{S}$, and their phonon spectra are known, i.e.,

$$
\begin{aligned}
& H_{M}=\sum_{j} \hbar \omega_{j} a_{j}^{+} a_{j}, \\
& H_{S}=\sum_{\mathbf{k}} \hbar \omega_{\mathbf{k}} b_{\mathbf{k}}^{+} b_{\mathbf{k}} .
\end{aligned}
$$

Here, $\omega_{j}$ are the frequencies of the nanoparticle modes with $a_{j}$ and $a_{j}^{+}$being the corresponding annihilation and creation operators; $\omega_{\mathbf{k}}$ are the frequencies of the substrate vibration modes of wave vectors $\mathbf{k}$ and $b_{\mathbf{k}}$ and $b_{\mathbf{k}}^{+}$are the corresponding phonon annihilation and creation operators. We have omitted the constant terms as they do not contribute to the dynamics of the system. Here, we consider a single phonon branch without loosing generality, but the formalism can be extended to include multiple branches. The interaction Hamiltonian $H_{M S}$ is also assumed to be quadratic in phonon operators,

$$
H_{M S}=\sum_{\mathbf{k}, j} \hbar\left(W_{\mathbf{k} j} b_{\mathbf{k}}^{+} a_{j}+\text { H.c. }\right),
$$

with $W_{\mathbf{k} j}$ being the coupling coefficient which is a function of $\omega_{\mathbf{k}}$ and $\omega_{j}$ and has the dimesion of angular frequency. We disregard the double annihilation and double creation of phonons in the present work.

Here, we consider two types of coupling. The first one is the Lorentzian coupling in which the coupling coefficient $W_{\mathbf{k} j}$ is a Lorentzian with its peak located at $\omega_{j}$ and has a width $\Gamma_{j}$. As long as the coupling between the nanoparticle and the substrate is weak, $W_{\mathbf{k} j}$ will be a peaked function of $\omega_{\mathbf{k}}$ and a separate peak will be present around each $\omega_{j}$. De- pending on the strength of the interaction, the sharpness of the peaks and the overlap between the neighboring peaks will differ. If the coupling is weak enough, we may neglect the overlaps, namely,

$$
W_{\mathbf{k} l}^{*} W_{\mathbf{k} j} \rightarrow\left|W_{\mathbf{k} l}\right|^{2} \delta_{l, j}
$$

For Lorentzian coupling, we assume that the coupling terms of different modes of the nanoparticle do not overlap, and hence, we can treat each nanoparticle mode separately.

For the second type of coupling, we consider the coupling coefficients which scale inversely as the square root of the product of the frequencies of the coupled modes, i.e.,

$$
W_{\mathbf{k} j}=\alpha\left(\omega_{\mathbf{k}} \omega_{j}\right)^{-1 / 2} .
$$

The coefficient $\alpha$ stands for the strength of the coupling and will depend on the interaction between the nanoparticle and the substrate. In both coupling types, $W_{\mathbf{k} j}$ is a function of $\omega_{\mathbf{k}}$ and $\omega_{j}$, explicit dependence on the wave vectors is not included for the sake of simplicity. The effect of initial temperatures of the parties, besides from the effect of temperature difference, is another major topic in its own and we leave that discussion to another paper. In the present paper, we consider the initial temperatures to be zero and limit our attention to the near-equilibrium case in the weak coupling regime. Strong coupling regime and nonequilibrium cases will also be treated separately.

\section{THEORETICAL METHODS}

\section{A. Equation of motion technique}

The time dependent occupancies of the nanoparticle modes can be obtained using Heisenberg's equation of motion, namely, $\dot{A}(t)=i[H, A(t)] / \hbar$. The equations of motion for the phonon annihilation operators are

$$
\begin{gathered}
\dot{a}_{l}(t)=-i \omega_{l} a_{l}(t)-i \sum_{\mathbf{k}} W_{\mathbf{k} l}^{*} b_{\mathbf{k}}(t), \\
\dot{b}_{\mathbf{k}}(t)=-i \omega_{\mathbf{k}} b_{\mathbf{k}}(t)-i \sum_{j} W_{\mathbf{k} j} a_{j}(t),
\end{gathered}
$$

that is, we have coupled differential equations for each operator. Performing Laplace transformation to both equations, a pair of coupled algebraic equations is obtained which can be decoupled algebraically, and by inverse transformation, the time dependent operator $a_{l}(t)$ is obtained as

$$
a_{l}(t)=\frac{a_{l}(0)}{2 \pi i} \oint_{B} \frac{e^{s t} d s}{s+i \omega_{l}+I_{l}(s)}-\frac{1}{2 \pi} \oint_{B} \frac{e^{s t} J_{l}(s) d s}{s+i \omega_{l}+I_{l}(s)} .
$$

where the integrals are to be evaluated along the Bromwich contour, with $I_{l}(s)$ and $J_{l}(s)$ being the substrate and interaction specific functions (see Appendix A).

The first and second terms in Eq. (9) stand for the contributions from the initial excitation of the nanoparticle and the initial temperature of the substrate, respectively. The second term does not contribute to the time dependent occupations of the nanoparticle mode, since the initial temperature of the substrate is considered to be zero. 


\section{B. Fano-Anderson method}

Since the Hamiltonian is quadratic in operators, its solution is equivalent to diagonalizing a matrix. Exact diagonalization of such quadratic Hamiltonians was shown to be possible by Fano $^{25}$ and Anderson ${ }^{26}$ independently, and the procedure is widely used in atomic physics, solid state physics, quantum optics, etc. Here, we will apply their method to the problem of phononic dissipation.

Solution of the Hamiltonian is equivalent to finding the dressed operators $\alpha\left(\omega_{\mathbf{q}}\right)$ such that the Hamiltonian is diagonal in terms of the dressed operators, $H$ $=\Sigma_{\mathbf{q}} \hbar \omega_{\mathbf{q}} \alpha^{+}\left(\omega_{\mathbf{q}}\right) \alpha\left(\omega_{\mathbf{q}}\right)$.

As described in AppendixB, one can write the time dependent occupancy of $j$ th mode as

$$
\begin{aligned}
\left\langle n_{j}(t)\right\rangle= & \left.\left.\left\langle n_{j}(0)\right\rangle\left|\int d \omega_{\mathbf{q}} g\left(\omega_{\mathbf{q}}\right)\right| \mu\left(\omega_{\mathbf{q}}, \omega_{j}\right)\right|^{2} e^{-i \omega_{\mathbf{q}} t}\right|^{2} \\
& +\int d \omega_{\mathbf{k}} g\left(\omega_{\mathbf{k}}\right)\left\langle n_{\mathbf{k}}(0)\right\rangle \\
& \times\left|\int d \omega_{\mathbf{q}} g\left(\omega_{\mathbf{q}}\right) \mu^{*}\left(\omega_{\mathbf{q}}, \omega_{j}\right) \nu^{*}\left(\omega_{\mathbf{q}}, \omega_{\mathbf{k}}\right) e^{-i \omega_{\mathbf{q}} t}\right|^{2},
\end{aligned}
$$

where $\mu\left(\omega_{\mathbf{q}}, \omega_{j}\right)$ are the expansion coefficients for the molecular operator $a_{j}$ in terms of the dressed operators $\alpha\left(\omega_{\mathbf{q}}\right)$ and $\nu\left(\omega_{\mathbf{q}}, \omega_{\mathbf{k}}\right)$ are that of the substrate operator $b_{\mathbf{k}}$ (see AppendixB).

Due to the finite range of substrate density of states (DOS) $g\left(\omega_{\mathbf{k}}\right)$, the integrals involved in the FA method are bounded. The method allows us to perform calculations for any $g\left(\omega_{\mathbf{k}}\right)$ and for any type of coupling with a single nanoparticle mode. The time dependent occupation is again separable as contributions from the initial temperature of the nanoparticle and that of the substrate. However, it should be noted that the FA method is applicable for any coupling type and any density of states for the substrate as long as we consider a single nanoparticle mode.

\section{Green's function method}

The effect of neighboring modes of nanoparticle having multimodes cannot be resolved within the above methods. That is, EoM and FA methods are restricted to the linear response regime. We use a more generalized method by which one can consider effects of neighboring modes. For this purpose, we employ Green's functions. Initially, the substrate temperature is zero and the phonon modes of the nanoparticle are empty except for the excitations which do not necessarily obey Bose-Einstein distribution. That is, the initial occupation of a nanoparticle mode is not a function of temperature. Therefore, we make use of zero temperature Green's functions instead of Matsubara formalism,

$$
d\left(j, t-t^{\prime}\right)=-i\left\langle T_{t} \hat{a}_{j}(t) \hat{a}_{j}^{+}\left(t^{\prime}\right)\right\rangle,
$$

$$
D\left(\mathbf{k}, t-t^{\prime}\right)=-i\left\langle T_{t} \hat{b}_{\mathbf{k}}(t) \hat{b}_{\mathbf{k}}^{+}\left(t^{\prime}\right)\right\rangle,
$$

where $T_{t}$ is the time-ordering operator, and the operators in Heisenberg picture are distinguished by a hat.

Since each term in the interaction Hamiltonian includes odd number of nanoparticle operators, only the even order terms contribute in the expansion. The first contribution due to the interaction is the second order term,

$$
d^{(0)}\left(\omega_{j}\right)^{2} \sum_{\mathbf{k}} W_{j \mathbf{k}}^{2} D^{(0)}\left(\omega_{\mathbf{k}}\right)=d^{(0)}\left(\omega_{j}\right)^{2} \Sigma^{(2)}\left(\omega_{j}\right),
$$

with $\Sigma^{(2)}\left(\omega_{j}\right)$ being the second order self-energy.

First, we wish to limit our attention to the case of a nanoparticle having a single mode. In obtaining the solution for a single mode, we will relate it to the FA result for the sake of illustration and then we will generalize our solution for the case of a nanoparticle having multiple modes. In doing so, we will be able to take the interplay between neighboring modes during dissipation into account.

For a single nanoparticle mode, the higher order terms can be expressed in terms of the second order self-energy and the free $\mathrm{GF}$ as

$$
d\left(\omega_{j}, \omega_{\mathbf{q}}\right)=d^{(0)}\left(\omega_{j}, \omega_{\mathbf{q}}\right)\left(1+d^{(0)} \Sigma^{(2)}+\left(d^{(0)} \Sigma^{(2)}\right)^{2}+\cdots\right) .
$$

For weak coupling, the above series can be written as

$$
d\left(\omega_{j}, \omega_{\mathbf{q}}\right)=\frac{d^{(0)}\left(\omega_{j}, \omega_{\mathbf{q}}\right)}{1-d^{(0)}\left(\omega_{j}, \omega_{\mathbf{q}}\right) \Sigma^{(2)}\left(\omega_{j}, \omega_{\mathbf{q}}\right)},
$$

hence, the retarded GF becomes

$$
d^{R}\left(\omega_{j}, \omega_{\mathbf{q}}\right)=\frac{1}{\omega_{\mathbf{q}}-\omega_{j}-\Sigma^{(2)}\left(\omega_{j}, \omega_{\mathbf{q}}\right)} .
$$

The real and imaginary parts of the second order selfenergy can be separated,

$$
\Sigma^{(2)}\left(\omega_{j}, \omega_{\mathbf{q}}\right)=\mathbb{P} \int \frac{d \omega_{\mathbf{k}} g\left(\omega_{\mathbf{k}}\right) W_{j \mathbf{k}}^{2}}{\omega_{\mathbf{q}}-\omega_{\mathbf{k}}}-i \pi g\left(\omega_{\mathbf{q}}\right) W_{j \mathbf{q}}^{2},
$$

where $\mathrm{P}$ is for principal part of the integral, and the spectral function is obtained by

$$
A\left(j, \omega_{\mathbf{q}}\right)=\frac{-2 \operatorname{Im} \Sigma^{(2)}\left(j, \omega_{\mathbf{q}}\right)}{\left(\omega_{\mathbf{q}}-\omega_{j}-\operatorname{Re} \Sigma^{(2)}\left(j, \omega_{\mathbf{q}}\right)\right)^{2}+\left(\operatorname{Im} \Sigma^{(2)}\left(j, \omega_{\mathbf{q}}\right)\right)^{2}} .
$$

The real part of the second order self-energy $\Sigma^{(2)}$ is equal to the shift in the $j$ th mode of the nanoparticle, $\sigma_{j}$, obtained previously using the FA method, and the square of the imaginary part of $\Sigma^{(2)}$ is $\left[\pi g\left(\omega_{\mathbf{q}}\right) W_{\mathbf{q} j}\right]^{2}$. That is, the FA expansion coefficient $\mu$ finds its expression in terms of the spectral function as

$$
\left|\mu\left(\omega_{\mathbf{q}}, \omega_{j}\right)\right|^{2}=\frac{A\left(j, \omega_{\mathbf{q}}\right)}{2 \pi g\left(\omega_{\mathbf{q}}\right)} .
$$

The time dependent GF can be written in terms of the spectral function and the time dependent occupancy of the $j$ th mode is obtained as 


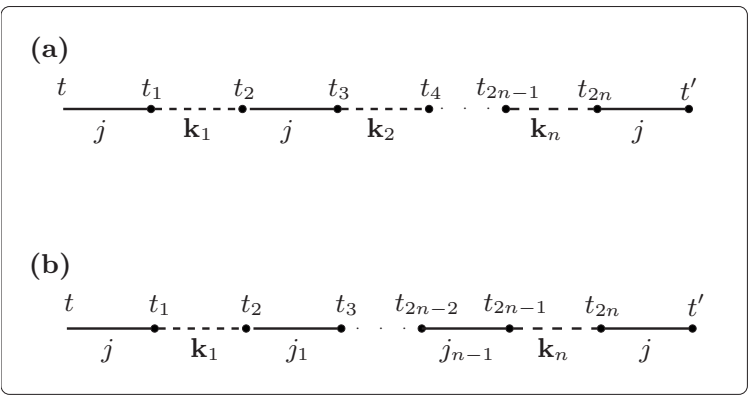

FIG. 2. Diagrams of order $2 n$. Solid lines are the phonon lines of the nanoparticle where the dashed lines are that of the substrate. (a) Diagram for the case of single nanoparticle mode $j . \mathbf{k}_{i}$ stand for the substrate modes. (b) Diagram of order $2 n$ when there exists multiple modes $\left(j_{i}\right)$ for the nanoparticle.

$$
\left\langle n_{j}(t)\right\rangle=\left\langle n_{j}(0)\right\rangle\left|\int \frac{d \omega_{\mathbf{q}}}{2 \pi} A\left(j, \omega_{\mathbf{q}}\right) e^{-i \omega_{\mathbf{q}} t}\right|^{2} .
$$

In order to incorporate the effect of neighboring modes, we follow the diagrammatic technique. As long as the Hamiltonian is quadratic, the primitive vertex, from which all diagrams are to be constructed, will consist of two phonon lines. That is, each interaction point is the intersection of two phonon lines. Since the interaction Hamiltonian relates a nanoparticle mode to a substrate mode only, each vertex contains one nanoparticle phonon line and a substrate phonon line. So the diagram of any order can be constructed [see Fig. 2(b)]. Having obtained the diagrammatic expansion for any order $2 n$, under certain conditions about the coupling type, the $(2 n)$ th order self-energy term can be expressed in terms of the second order term and the free GF of the nanoparticle modes. If the fraction $W_{\mathbf{k}_{1} j_{1}} / W_{\mathbf{k}_{1} j_{2}}$ is independent of $\mathbf{k}_{1}$, the self-energy for the multimode case can be found exactly where the fourth and sixth order contributions can be written as

$$
\begin{aligned}
& \Sigma^{(4)}\left(j, \omega_{q}\right)=\frac{1}{W_{\mathbf{q} j}^{2}}\left(\sum_{j_{1}} d^{(0)}\left(j_{1}, \omega_{q}\right) W_{\mathbf{q} j_{1}}^{2}\right)\left(\Sigma^{(2)}\left(j, \omega_{\mathbf{q}}\right)\right)^{2}, \\
& \Sigma^{(6)}\left(j, \omega_{q}\right)=\frac{1}{W_{\mathbf{q} j}^{4}}\left(\sum_{j_{1}} d^{(0)}\left(j_{1}, \omega_{q}\right) W_{\mathbf{q} j_{1}}^{2}\right)^{2}\left(\Sigma^{(2)}\left(j, \omega_{\mathbf{q}}\right)\right)^{3} .
\end{aligned}
$$

By mathematical induction, the $(2 n)$ th term is found as

$$
\Sigma^{(2 n)}\left(j, \omega_{q}\right)=\frac{1}{W_{\mathbf{q} j}^{2(n-1)}}\left(\sum_{j_{1}} d^{(0)}\left(j_{1}, \omega_{q}\right) W_{\mathbf{q} j_{1}}^{2}\right)^{n-1}\left(\Sigma^{(2)}\left(j, \omega_{\mathbf{q}}\right)\right)^{n}
$$

and hence,

$$
\Sigma\left(j, \omega_{\mathbf{q}}\right)=\frac{\Sigma^{(2)}\left(j, \omega_{\mathbf{q}}\right)}{1-\frac{\Sigma^{(2)}\left(j, \omega_{\mathbf{q}}\right)}{W_{j \mathbf{q}}^{2}} \sum_{j^{\prime} \neq j} W_{j^{\prime} \mathbf{q}}^{2} d^{(0)}\left(j^{\prime}, \omega_{\mathbf{q}}\right)} .
$$

Once the self-energy is found, the spectral function, therefore the time dependent occupancy of the nanoparticle modes can be calculated.

A few remarks about the above expression for the selfenergy follow. First, it is exact in the sense that it includes contributions from diagrams to all orders. Second $\Sigma\left(j, \omega_{\mathbf{q}}\right)$ is not a quadratic function of coupling $W_{\mathbf{k} j}$ anymore, as it was in the single mode approximation case. Rather, the decay rate of $j$ th mode collects contributions from all other modes also. Third, the spectral function is not of Lorentzian shape anymore; extra peaks and dips in the spectral function are in question which will be analyzed numerically in the following section.

\section{RESULTS AND DISCUSSIONS}

In this section, we will apply the above methods using Lorentzian and square-root coupling to analyze the dependence of the decay rate on the properties of nanoparticles and substrates having one-dimensional (1D) and twodimensional (2D) Debye DOSs. The analytical results obtained using EoM technique and numerical results obtained using FA and GF methods are discussed separately. An application for a real physical system using DFT and the GF method will also be presented.

(i) As an application of the EoM method, we consider the nanoparticle that has a single mode coupled to a $1 \mathrm{D}$ or $2 \mathrm{D}$ Debye substrate. The substrate is initially at zero temperature. Assuming Lorentzian coupling, namely,

$$
W_{\mathbf{k} j}^{2}=\frac{\alpha^{2} \Gamma}{2 \pi} \frac{1}{\left(\omega_{\mathbf{k}}-\omega_{j}\right)^{2}+\Gamma^{2} / 4},
$$

we have for $I_{j}(s)$ (see Appendix A)

$$
I_{j}=\frac{\alpha^{2} \Gamma c_{D}}{2 \pi i} \int_{0}^{\omega_{\max }} \frac{d \omega_{\mathbf{k}} \omega_{\mathbf{k}}^{d-1}}{\left(\omega_{\mathbf{k}}-i s\right)\left(\omega_{\mathbf{k}}-\omega_{j}-i \frac{\Gamma}{2}\right)\left(\omega_{\mathbf{k}}-\omega_{j}+i \frac{\Gamma}{2}\right)},
$$

where $d$ is the dimension of the substrate, $c_{D}$ is the corresponding Debye constant for DOS, and $g_{d}(\omega)=c_{D} \omega^{d-1} . \omega_{j}$ and $\Gamma$ are real and positive, and by definition of Laplace transformation, $\operatorname{Re}(s)>0$. In the weak coupling regime, the width of the Lorentzian will be much smaller than $\omega_{j}$ and $\omega_{\max },{ }^{28}$ so we can approximate the above integral by extending the limits of integration to $(-\infty, \infty)$, in which case the integral can be evaluated analytically on the complex $\omega_{\mathbf{k}}$ plane with the result $I_{j}(s)=\alpha^{2} c_{D}\left(\omega_{j}-i \Gamma / 2\right)^{d-1} /\left(s+i \omega_{j}\right.$ $+\Gamma / 2)$. Performing the inverse transformation, one finds

$$
\left\langle n_{j}(t)\right\rangle=\left\langle n_{j}(0)\right\rangle \frac{e^{-\Gamma t / 2}}{4|\Delta|^{2}}\left|(\Gamma+\Delta) e^{\Delta t / 2}-(\Gamma-\Delta) e^{-\Delta t / 2}\right|^{2},
$$

where $\Delta^{2}=\Gamma^{2} / 4-4 \alpha^{2} c_{D}\left(\omega_{j}-i \Gamma / 2\right)^{d-1}$. 


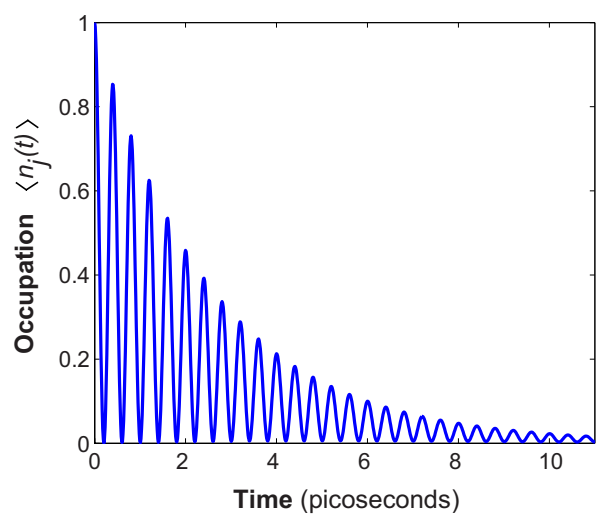

FIG. 3. (Color online) Decay of a single nanoparticle mode $j$ coupled to a 2D-Debye substrate. The coupling is Lorentzian. Occupation $\left\langle n_{j}(t)\right\rangle$ at time $t$ is given relative to the initial occupation $\left\langle n_{j}(0)\right\rangle$.

It is noted that the domain of applicability of the EoM technique is quite limited due to the fact that the inverse Laplace transformation is not always possible neither analytically nor numerically. Nevertheless, in certain cases, the EoM method enables us to get analytical results within some approximations. Figure 3 shows the decay of a single nanoparticle mode to a 2D-Debye substrate obtained by the EoM method. The oscillatory behavior is due to the splitting of the molecular spectrum into two.

(ii) Next, using FA and GF methods, we discuss the critical features in the dissipation of single mode energies of nanoparticle to $1 \mathrm{D}$ and $2 \mathrm{D}$ substrates. In the weak coupling regime, the width of the spectral function $A\left(j, \omega_{\mathbf{q}}\right)$ will be small compared to $\omega_{\mathbf{q}}$, provided that $\omega_{j}$ is not close to zero, which is already satisfied for nanoparticles. In this limit, the imaginary part of the self-energy can be interpreted as twice the decay rate, $\gamma_{j}=\operatorname{Im} \Sigma\left(j, \omega_{\mathbf{q}}\right) / 2$. Therefore, the dependence of decay rate on the interaction type and strength as well as on the frequency of the nanoparticle can be obtained from the spectral function.

For the case of Lorentzian coupling, the interaction strength $\alpha$ is linear with $W_{\mathbf{k} j}$, which shows that the decay rate increases with $\alpha^{2}$. If the coupling is only a function of the distance between interacting atoms of the substrate and the nanoparticle, the coupling has the form of Eq. (6) with $\alpha$ being proportional to a spring constant $k_{\text {int }}$ connecting the interacting atoms. Since the spectral function scales with $\alpha^{2}$, decay rate increases with $k_{i n t}^{2}$ for inverse-square-root coupling case. The $k_{\text {int }}^{2}$ law was previously obtained using elastic continuum model for phononic dissipation in physisorption systems. ${ }^{27}$

The dependence on the nanoparticle mode frequency is a key issue we wish to emphasize in phononic energy dissipation. In Lorentzian coupling case, the decay rate is determined by the width of the Lorentzian rather than the frequency. On the other hand, for inverse-square-root coupling [see Eq. (6)], it is inversely proportional to the nanoparticle mode frequency $\omega_{j}$. It is evident from Eqs. (B12) and (18) that phonons in mode $\omega_{j}$ decay faster as the substrate DOS at the center of the peak, $\omega_{j}-\operatorname{Re} \Sigma\left(j, \omega_{\mathbf{q}}\right)$, increases. A crucial consequence of dependence on substrate DOS is that if the DOS at the peak of the spectral function tends to zero, the spectral function (and $\left|\mu\left(\omega_{\mathbf{q}}, \omega_{j}\right)\right|^{2}$ ) has the form of a $\delta$ function. In the language of dressed modes, this corresponds to a localized mode, i.e., it does not decay at all. Such localized states are also known to occur in, e.g., solid state physics ${ }^{26}$ and atomic physics. ${ }^{25}$ For weak coupling, the real part of the self-energy is small, so the peak of the spectral function is not altered significantly from its original position $\omega_{j}$. In other words, lying outside the continuum of substrate modes, it is unlikely to be shifted into the range where it can decay or vice versa. Decay of such localized modes is possible, on the other hand, by including the anharmonic terms in the Hamiltonian. Localized modes of the harmonic approximation now will gain finite width due to multiphonon interactions. In general, three-phonon interactions are weak, four-phonon processes are even weaker, and the first nonzero contribution from three-phonon processes arises at the second-order term of the diagramatic expansion. Another mechanism for decay of localized harmonic modes can be due to double annihilation and creation terms in the interaction Hamiltonian which are neglected in this study [see Eq. (4)]. Namely, though localized modes are not truly localized considering anharmonic terms or double annihilation/creation terms, their decay rates will be small. Another important effect about DOS dependence takes place when the spectral peak coincides with a van Hove singularity of the substrate DOS, by which the decay rate is enhanced abruptly.

(iii) We also investigate the effect of a neighboring mode within square-root coupling in 1D and 2D Debye substrate densities of states using GF method. We consider four nanoparticle modes, $\omega_{1}=0.7 \omega_{\max }, \omega_{2}=0.65 \omega_{\max }, \omega_{3}=0.55 \omega_{\max }$, and $\omega_{4}=0.45 \omega_{\max }$. The effect is analyzed pairwise, namely, we consider $\left(\omega_{1}, \omega_{2}\right),\left(\omega_{1}, \omega_{3}\right)$, and $\left(\omega_{1}, \omega_{4}\right)$ as the nanoparticle modes, keeping other parameters unchanged. That is, we keep $\omega_{1}$ constant while changing the second mode and investigate dependence of decay of $\omega_{1}$ mode on the separation from the second nanoparticle mode. For both 1D-Debye [Figs. 4(a)-4(c)] and 2D-Debye [Figs. 4(d)-4(f)], cases, we observe that the decay of excited modes gain a retardation as the mode frequencies get closer. A second behavior is the enhancement of fluctuations during decay as the mode frequencies get closer. Both behaviors can be understood in terms of the spectral functions. In Fig. 5(a), spectral functions of $\omega_{1}$ and $\omega_{3}$ modes are plotted for single mode (dashed curves) and multimode (solid curves) obtained using GF calculations. It is seen that the overlap is negligible and the spectra are not changed considerably. When the modes are closer [Fig. 5(b)], the single-mode spectra (dashed curves) have finite overlap; correspondingly, the multimode spectral functions affect each other. The Lorentzian shape is distorted and the peak of $\omega_{2}$ mode is enhanced. These result in retardation and fluctuations during decay. More precisely, the finite overlap of spectra allows the nanoparticle to gain phonons back which are previously discharged to the substrate. This phonon exchange process continues during the dissipation and gives rise to retardations and fluctuations observed in Fig. 4.

(iv) In order to provide a comparison of the results obtained from the quantum treatment with those obtained by 

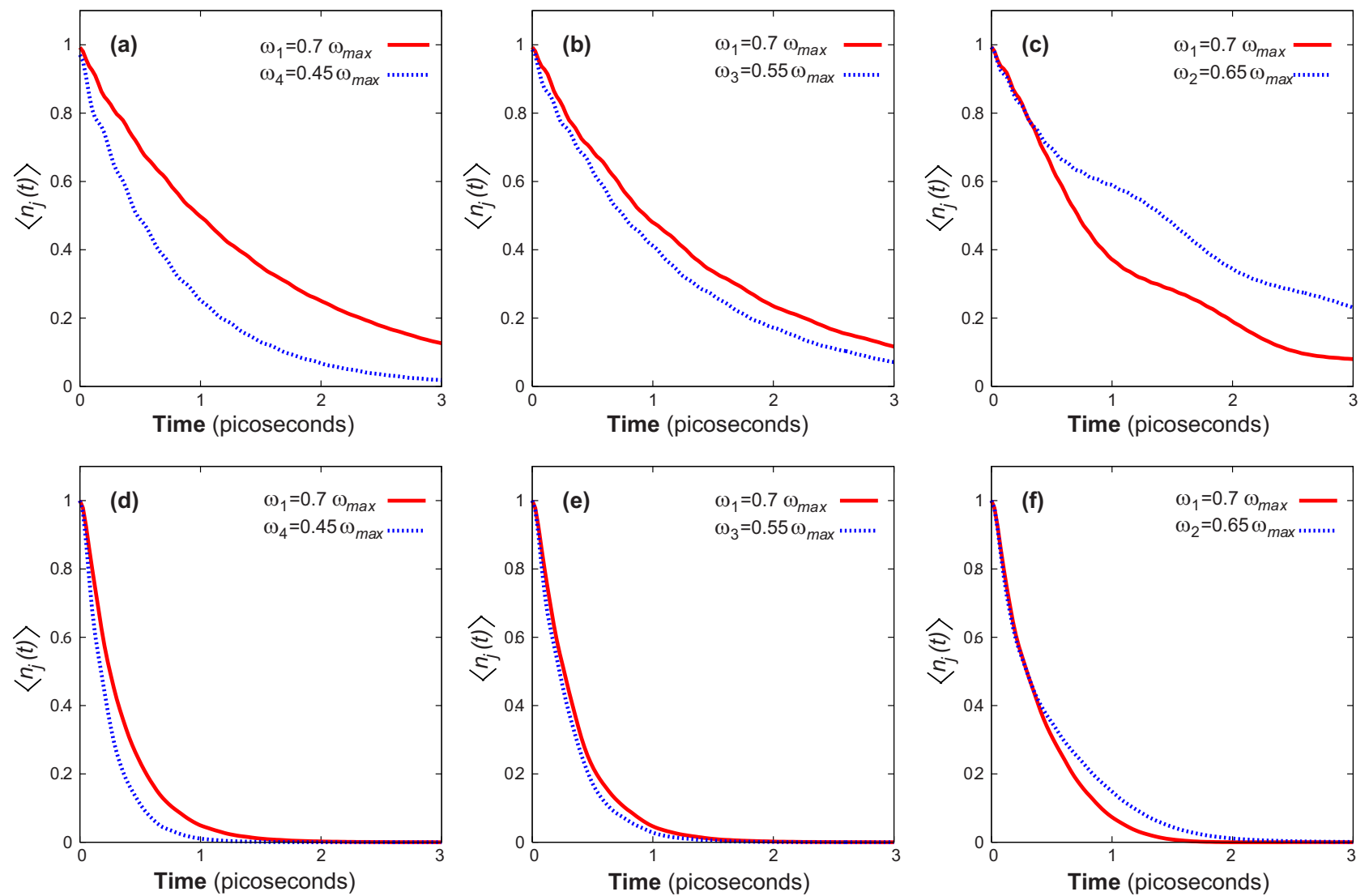

FIG. 4. (Color online) Effect of neighboring modes. (a)-(c) are for 1D-Debye DOS and (d)-(f) are for 2D-Debye DOS with nanoparticle vibration frequencies $\omega_{1}=0.7 \omega_{\max }, \omega_{2}=0.65 \omega_{\max }, \omega_{3}=0.55 \omega_{\max }$, and $\omega_{4}=0.45 \omega_{\max }$. (a) and (d), (b) and (e), and (c) and (f) show dissipation of phonon occupation for the pairs $\left(\omega_{1}, \omega_{4}\right),\left(\omega_{1}, \omega_{3}\right)$, and $\left(\omega_{1}, \omega_{2}\right)$, respectively.

classical treatment, we carry out calculations using classical molecular dynamics method. We use a simple but effective approach, where we consider the nanoparticle (substrate) as a cluster (lattice) of masses and harmonic springs in the first nearest neighbor approximation, and with the lattice having

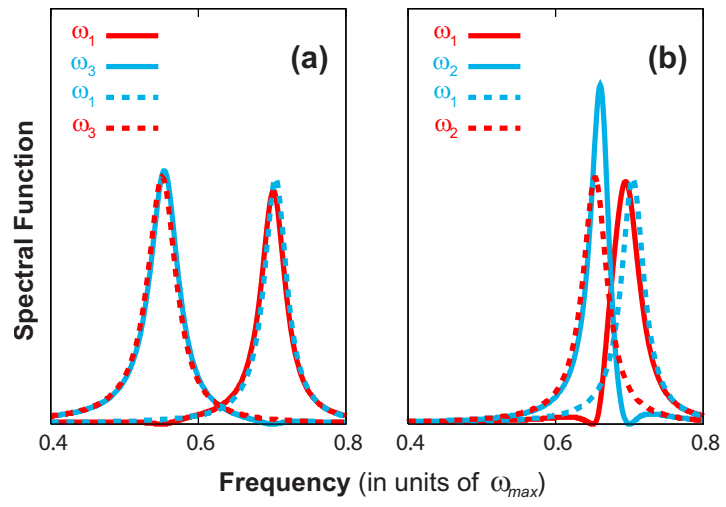

FIG. 5. (Color online) Effect of a neighboring mode on the spectral function. Dashed curves are the single mode spectral functions, whereas the solid curves are spectral functions in the existence of a neighboring mode. (a) Spectra of $\omega_{1}=0.7 \omega_{\max }$ and $\omega_{3}$ $=0.55 \omega_{\max }$ for both cases are almost the same. (b) Spectra of $\omega_{1}$ $=0.7 \omega_{\max }$ and $\omega_{2}=0.65 \omega_{\max }$ get narrowed and distorted when single mode condition is relaxed. different dimensionalities. The interaction is described by a harmonic spring between an atom of the nanoparticle and a substrate atom. Using the dynamical matrix, the eigenmodes of the isolated nanoparticle are determined and the initial energy is loaded to the desired modes by giving the initial velocities to the atoms in correspondence with the modes. In the presence of the interaction between the nanoparticle and the substrate, the differential equations and hence the motion of atoms are determined in discrete time steps which are on the order of femtoseconds. Since the classical version of the problem, which is stated above, is not an exact analog to the quantum one and due to quantum versus classical natures of the two, we compare and contrast the basic features of the results emerging from them.

In agreement with the earlier prediction based on the elastic continuum model ${ }^{27}$ and with the result previously obtained using the single mode, the dependence of decay rate on the interaction strength obeys $k_{i n t}^{2}$ law for weak coupling. Likewise, the dependence on vibrational mode frequency verifies the previously obtained result, namely, while keeping the coupling strength constant, higher frequency modes decay slower. We should note that the substrate DOS in the neighborhood of the nanoparticle mode frequency also affects the decay rates. Using a 1D substrate and choosing the nanoparticle modes away from the maximum frequency of the substrate, the effect of substrate DOS is minimized. Although the density of substrate phonon modes is higher for 
higher frequencies, the decay rate decreases due to nanoparticle mode frequency dependence.

Another property of the dissipation process becomes apparent when the dynamics is analyzed for a nanoparticle having one and two vibrational modes. We consider a diatomic molecule and a linear triatomic molecule, which have one and two vibrational modes along the molecular axis, where the interaction is also along the molecular axis. The effect of neighboring mode can be be analyzed by setting one of the modes of the triatomic molecule at the same frequency with the frequency of the diatomic one. Exciting only the common frequency of both diatomic and triatomic molecules, we compare the decay rates, keeping the interaction and substrate parameters fixed. In the weak coupling regime, it is observed that the decay rate of the common mode does not change appreciably. Moreover, exciting both vibrational modes of the triatomic molecule does not effect the decay rate of the common mode to a great extend. This property becomes more apparent when the coupling strength is weakened. Since the vibrational modes of a triatomic molecule along the molecular axis are well separated, this result is expected in the light of GF solution of the quantum Hamiltonian. The mode localization effect is also tested using classical MD simulations. Unlike the quantum solution, a molecular mode whose frequency lies above the maximum frequency of the substrate has a small but yet finite decay rate.

(v) Finally, we present a specific and more realistic study of energy dissipation from the excited modes of a benzene $\left(\mathrm{C}_{6} \mathrm{H}_{6}\right)$ molecule coupled to graphene using GF method. Here, the multimode frequencies of $\mathrm{C}_{6} \mathrm{H}_{6}$, the continuous phonon spectrum of graphene, and the coupling between them are calculated by using first-principles ultrasoft pseudopotential ${ }^{29}$ plane-wave method ${ }^{30,31}$ within DFT. ${ }^{32}$ The exchange correlation potential has been approximated by generalized gradient approximation ${ }^{33}$ using PW91 functional.

These calculations allow an accurate quantum mechanical treatment. All atomic positions are optimized by the conjugate gradient method and the system is considered to be at equilibrium when Hellman-Feynman forces are below $10 \mathrm{meV} / \AA$. A large supercell is used for the free $\mathrm{C}_{6} \mathrm{H}_{6}$ molecule so that the distance to the nearest atom of the neighboring $\mathrm{C}_{6} \mathrm{H}_{6}$ molecule is above $10 \AA$. A plane-wave basis set with kinetic energy cutoff $\hbar^{2}|\mathbf{k}+\mathbf{G}|^{2} / 2 m=350 \mathrm{eV}$ has been used. Each atom is shifted by $0.01 \AA$ in each direction from their equilibrium positions, and the resulting forces on each atom are used to construct the dynamical matrix such that

$$
K_{\alpha \beta}^{\mu \nu}=\frac{1}{2} \frac{F_{\alpha \beta}^{\mu \nu+}-F_{\alpha \beta}^{\mu \nu-}+F_{\beta \alpha}^{\nu \mu+}-F_{\beta \alpha}^{\nu \mu-}}{2 d},
$$

where $F_{\alpha \beta}^{\mu \nu \pm}$ denotes the force on atom $\beta$ along $\nu$ when $\alpha$ th atom is moved along $\mu$ in positive or negative direction; $d$ is the displacement imposed on a specific atom. The dynamical matrix is defined in terms of these forces as $D_{i j}$ $=D_{3(\alpha-1)+\mu, 3 \beta-1+\nu}=K_{\alpha \beta}^{\mu \nu}$. In solving the dynamical matrix, the vibration frequencies and the corresponding normal coordinates are determined.

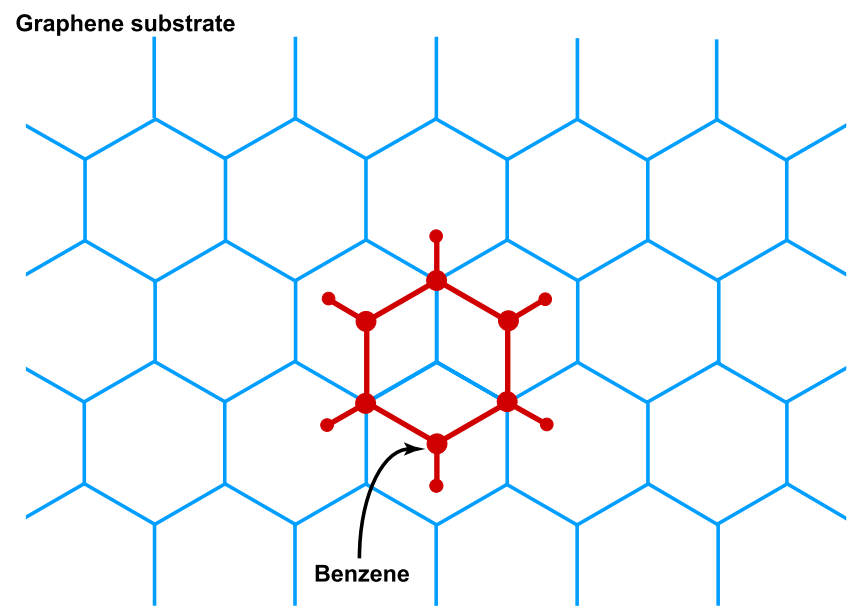

FIG. 6. (Color online) Relaxed geometry of benzene on graphene. Blue (gray) lines show the graphene structure.

The interaction between the $\mathrm{C}_{6} \mathrm{H}_{6}$ molecule and the underlying graphene is calculated by relaxing the geometry in a supercell of the same size used for free $\mathrm{C}_{6} \mathrm{H}_{6}$; the final geometry is schematically shown in Fig. 6. The equilibrium distance between the molecule and graphene is $3.75 \AA$; that is, the interaction is weak. For the sake of simplicity, we assume that the interaction between the molecule and the substrate is achieved by the $\mathrm{C}$ atoms lying on top of each other, and varying the benzene-graphene distance vertically, we obtain a total energy versus distance curve to which we perform a quadratic fit to calculate this effective interaction constant. The interaction constant between $\mathrm{C}$ atoms lying on top of each other is found to be $13.55 \mathrm{eV} / \AA^{2}$.

Since the interaction is weak and the $\mathrm{C}_{6} \mathrm{H}_{6}$-graphene distance is large, the dissipation will occur mostly through the out-of-plane motions of $\mathrm{C}_{6} \mathrm{H}_{6}$ atoms due to their coupling to the transverse modes of graphene. Using the normal coordinates of these vibrational modes, the contribution of each atom to the interaction can be determined. When a single atom of the molecule is interacting with the substrate, the coupling coefficient goes like $W_{\mathbf{k} j}=c \omega_{i n t}^{2} / \sqrt{\omega_{\mathbf{k}} \omega_{j}}$, where $\omega_{j}$ stands for the frequency of $j$ th mode of the molecule and $c$ is the normal coordinate of the interacting atom in $j$ th mode. When there are more than one atoms interacting with the substrate (as is the case for $\mathrm{C}_{6} \mathrm{H}_{6}$ graphene), we sum over those interacting degrees of freedom to find the effective coefficient $c$ which scales the coupling strength. The out-ofplane vibrational modes of benzene and the scaling coefficients are given in Table I. It is worth mentioning that $\mathrm{C}_{6} \mathrm{H}_{6}$ has doubly degenerate modes which is due to hexagonal symmetry of the molecule, i.e., $(1,2),(5,6)$, and $(7,8)$ of Table I form degenerate pairs for free $\mathrm{C}_{6} \mathrm{H}_{6}$. The coefficients of the degenerate modes are identical due to symmetry grounds.

The spectral function $A\left(j, \omega_{\mathbf{q}}\right)$ for each mode is calculated using the GF method. Since only the lowest six of the outof-plane mode frequencies lie within the range of transverse substrate phonons, they gain a finite width while the remaining three modes stay localized. The spectra of the lowest lying modes and the DOS of transverse substrate phonons 
TABLE I. The out-of-plane vibrational modes of $\mathrm{C}_{6} \mathrm{H}_{6}$ and the effective coefficients which scale the coupling strength.

\begin{tabular}{lcc}
\hline \hline & $\begin{array}{c}\text { Frequency } \omega_{j} \\
(\mathrm{THz})\end{array}$ & \\
\hline 1 & 11.99 & Effective coefficient $c_{j}$ \\
2 & 11.99 & 0.962 \\
3 & 20.58 & 0.962 \\
4 & 21.39 & 0.301 \\
5 & 26.03 & 1.105 \\
6 & 26.03 & 0.489 \\
7 & 29.66 & 0.489 \\
8 & 29.66 & 0.566 \\
9 & 30.12 & 0.566 \\
\hline \hline
\end{tabular}

are shown in Fig. 7. The coupling strengths of degenerate modes are equal; therefore, their spectral functions are identical. Dependence of the shift and broadening of the spectral function on the coupling coefficient manifests itself as the narrow spectral peak of the third mode when compared to
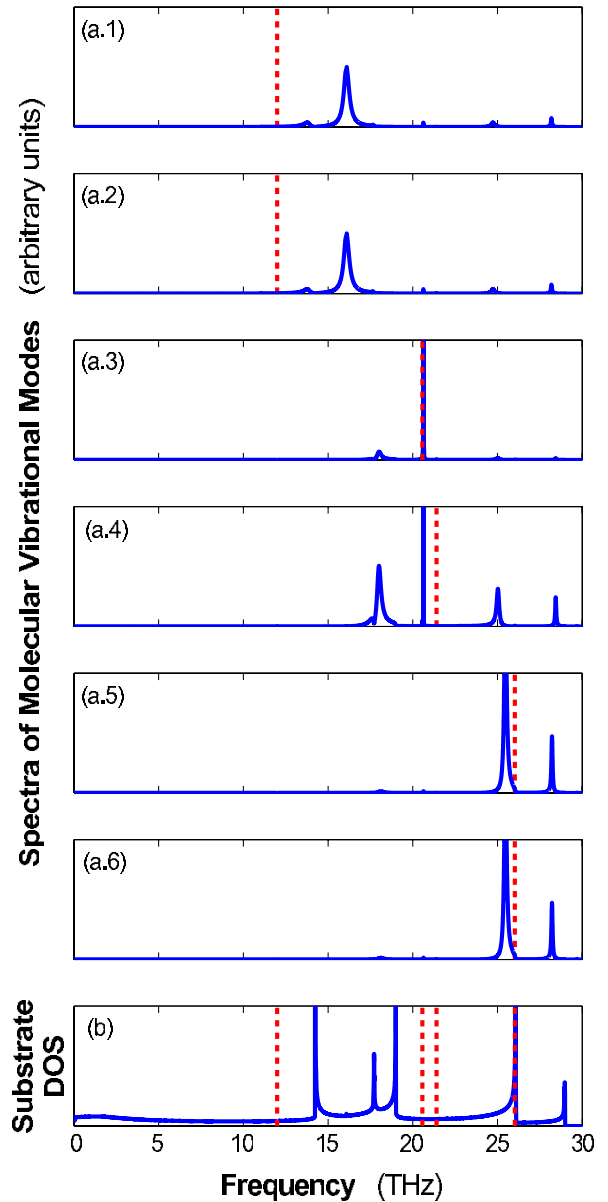

FIG. 7. (Color online) Spectra of the six lowest lying vibrational modes of benzene when interacting with a graphene sheet (a.1)(a.6) and DOS of transverse phonons of the graphene substrate (b). The red (dashed) lines indicate the vibrational frequencies of the free benzene molecule. that of the first two modes. Although the third and fourth modes are close to each other, the dissipation of the fourth one is much faster than that of the third mode. The broadening of the fifth and sixth modes would be much larger if their shifted peaks were matched the singularity of the DOS near $26 \mathrm{THz}$. However, they gain a shift which pushes the peak far from singularity. We observe that there might appear more than one peaks in the spectrum of a single mode. This is due to two reasons, the contributions from the singularities in the substrate DOS and the interaction stimulated anharmonicities within the molecule. Although the molecule is treated in the harmonic approximation, interaction Hamiltonian gives rise to an indirect coupling between different vibrational modes of the free molecule.

We note that the results for the specific case of benzene molecule weakly interacting with graphene are in agreement with the results obtained in part (iii). More specifically, dependence of spectral width on coupling strength, effect of van Hove singularities, and the interplay between neighboring modes are illustrated in this specific example.

\section{CONCLUSION}

The phononic dissipation from a nanostructure weakly coupled to a substrate has been analyzed using three different methods. The EoM technique is able to yield analytical results, but has a limited range of applicability because of the fact that inverse Laplace transformation is not always possible. On the other hand, FA diagonalization is possible for any type of substrate density of states and any type of coupling, but is restricted to considerations of single nanoparticle mode only. Using GFs, the effect of neighboring nanoparticle modes can also be investigated. It is found that the stronger the coupling is, the faster is the rate of dissipation. Since the width of the spectrum of a single nanoparticle mode scales with the value of the substrate DOS at the shifted frequency of the nanoparticle mode, we observe that a single nanoparticle mode coupled to a 2D-Debye substrate decays faster than the one coupled to a 1D-Debye substrate. This situation can be reversed for those frequencies for which 1D-DOS is higher than the 2D-DOS, namely, for low frequencies (larger nanoparticles). That is, at frequencies at which 1D-DOS has higher values than 2D-DOS, decay rate of a mode coupled to the $1 \mathrm{D}$ substrate will be higher than that of the mode coupled to $2 \mathrm{D}$ substrate, provided that the remaining factors are kept identical. The presence of neighboring nanoparticle modes affect each other's decay rate when their spectral functions have an appreciable overlap. Transitions between nanoparticle modes take place via the substrate modes; therefore, retardation as well as fluctuations become important when the modes are close enough. Furthermore, using the results obtained from a first-principles study, interplay between molecular modes depending on the substrate DOS is demonstrated.

\section{ACKNOWLEDGMENTS}

This work was supported by The Scientific and Technological Research Council of Turkey through Grant No. 
TBAG-104T537. R.T.S. acknowledges financial support from TÜBA-GEBIP. This research was supported in part by TÜBITAK through TR-Grid e-Infrastructure Project (http:// www.grid.org.tr).

\section{APPENDIX A: DETAILS OF EQUATION OF MOTION TECHNIQUE}

The Laplace transformed form of equations of motion are [see Eqs. (7) and (8)]

$$
\begin{gathered}
\bar{a}_{l}(s)\left(s+i \omega_{l}\right)=a_{l}(0)-i \sum_{\mathbf{k}} W_{\mathbf{k} l}^{*} \bar{b}_{\mathbf{k}}(s), \\
\bar{b}_{\mathbf{k}}(s)\left(s+i \omega_{\mathbf{k}}\right)=b_{\mathbf{k}}(0)-i \sum_{j} W_{\mathbf{k} j} \bar{a}_{j}(s),
\end{gathered}
$$

where $s$ is the Laplace frequency. Solving for $\bar{a}_{l}(s)$, one obtains

$$
\begin{aligned}
\bar{a}_{l}(s)= & \frac{a_{l}(0)}{s+i \omega_{l}}-i \sum_{\mathbf{k}} \frac{W_{\mathbf{k} l}^{*} b_{\mathbf{k}}(0)}{\left(s+i \omega_{\mathbf{k}}\right)\left(s+i \omega_{l}\right)} \\
& -\sum_{\mathbf{k} j} \frac{W_{\mathbf{k} l}^{*} W_{\mathbf{k} j} \bar{a}_{j}(s)}{\left(s+i \omega_{\mathbf{k}}\right)\left(s+i \omega_{l}\right)} .
\end{aligned}
$$

Considering the couplings to be nonoverlapping [see Eq. (5)], we are left with the relation

$$
\bar{a}_{l}(s)=\frac{a_{l}(0)}{s+i \omega_{l}+\sum_{\mathbf{k}} \frac{\left|W_{\mathbf{k} l}\right|^{2}}{s+i \omega_{\mathbf{k}}}}-i \frac{\sum_{\mathbf{k}} \frac{W_{\mathbf{k} l}^{*} b_{\mathbf{k}}(0)}{s+i \omega_{\mathbf{k}}}}{s+i \omega_{l}+\sum_{\mathbf{k}} \frac{\left|W_{\mathbf{k} l}\right|^{2}}{s+i \omega_{\mathbf{k}}}} .
$$

Having obtained $\bar{a}_{l}(s)$ in terms of $a_{l}(0)$ and $b_{\mathbf{k}}(0)$, the inverse Laplace transform will yield $a_{l}(t)$; thus, we can obtain the time dependent occupancy of the $l$ th mode.

We convert the summations into integrals over the substrate modes and denote them as

$$
\begin{gathered}
I_{l}(s)=\sum_{\mathbf{k}} \frac{\left|W_{\mathbf{k} l}\right|^{2}}{s+i \omega_{\mathbf{k}}}=\int \frac{d \omega_{\mathbf{k}} g\left(\omega_{\mathbf{k}}\right)\left|W_{\mathbf{k} l}\right|^{2}}{s+i \omega_{\mathbf{k}}}, \\
J_{l}(s)=\sum_{\mathbf{k}} \frac{W_{\mathbf{k} l}^{*} b_{\mathbf{k}}(0)}{s+i \omega_{\mathbf{k}}}=\int \frac{d \omega_{\mathbf{k}} g\left(\omega_{\mathbf{k}}\right) W_{\mathbf{k} l}^{*} b_{\mathbf{k}}(0)}{s+i \omega_{\mathbf{k}}},
\end{gathered}
$$

where $g\left(\omega_{\mathbf{k}}\right)$ is the phonon density of states for the substrate, $I_{l}$ and $J_{l}$ depend on $s$, and $J_{l}$ is an operator. We can write Eq. (A4) as

$$
\bar{a}_{l}(s)=\frac{a_{l}(0)}{s+i \omega_{l}+I_{l}(s)}-i \frac{J_{l}(s)}{s+i \omega_{l}+I_{l}(s)} .
$$

The inverse transform of $\bar{a}_{l}(s)$ is

$$
a_{l}(t)=\frac{a_{l}(0)}{2 \pi i} \oint_{B} \frac{e^{s t} d s}{s+i \omega_{l}+I_{l}(s)}-\frac{1}{2 \pi} \oint_{B} \frac{e^{s t} J_{l}(s) d s}{s+i \omega_{l}+I_{l}(s)} .
$$

\section{APPENDIX B: DETAILS OF FANO-ANDERSON METHOD}

Since the bare phonon operators $a_{j}$ and $b_{\mathbf{k}}$ form a complete set of operators for the combined system, the dressed operators $\alpha\left(\omega_{\mathbf{q}}\right)$ can be expanded in terms of the bare operators as

$$
\alpha\left(\omega_{\mathbf{q}}\right)=\sum_{j} \mu\left(\omega_{\mathbf{q}}, \omega_{j}\right) a_{j}+\sum_{\mathbf{k}} \nu\left(\omega_{\mathbf{q}}, \omega_{\mathbf{k}}\right) b_{\mathbf{k}},
$$

and they satisfy the eigenoperator equation $\left[\alpha\left(\omega_{\mathbf{q}}\right), H\right]$ $=\hbar \omega_{\mathbf{q}} \alpha\left(\omega_{\mathbf{q}}\right)$. Conversely, we find the bare operators by the following expressions in terms of the dressed operators:

$$
\begin{aligned}
& a_{j}=\sum_{\mathbf{q}} \mu^{*}\left(\omega_{\mathbf{q}}, \omega_{j}\right) \alpha\left(\omega_{\mathbf{q}}\right), \\
& b_{\mathbf{k}}=\sum_{\mathbf{q}} \nu^{*}\left(\omega_{\mathbf{q}}, \omega_{\mathbf{k}}\right) \alpha\left(\omega_{\mathbf{q}}\right) .
\end{aligned}
$$

Substituting Eq. (B1) into the eigenoperator relation, one ends up with a pair of equations,

$$
\begin{aligned}
& \mu\left(\omega_{\mathbf{q}}, \omega_{j}\right)\left(\omega_{\mathbf{q}}-\omega_{j}\right)=\sum_{\mathbf{k}} \nu\left(\omega_{\mathbf{q}}, \omega_{\mathbf{k}}\right) W_{\mathbf{k} j}, \\
& \nu\left(\omega_{\mathbf{q}}, \omega_{\mathbf{k}}\right)\left(\omega_{\mathbf{q}}-\omega_{\mathbf{k}}\right)=\sum_{j} \mu\left(\omega_{\mathbf{q}}, \omega_{j}\right) W_{\mathbf{k} j}^{*},
\end{aligned}
$$

which can be solved self-consistently to obtain $\omega_{\mathbf{q}}$. Using Eq. (B5), $\nu$ can be expressed in terms of $\mu$ as

$$
\nu\left(\omega_{\mathbf{q}}, \omega_{\mathbf{k}}\right)=\left(\frac{P}{\omega_{\mathbf{q}}-\omega_{\mathbf{k}}}+\delta\left(\omega_{\mathbf{q}}-\omega_{\mathbf{k}}\right) z\left(\omega_{\mathbf{q}}\right)\right) \sum_{j} \mu\left(\omega_{\mathbf{q}}, \omega_{j}\right) W_{\mathbf{k} j}^{*},
$$

where $\mathbb{P}$ stands for the principal part, and the $\delta$-function term accounts for the contribution from the singularity. Inserting Eq. (B6) into Eq. (B4), the following relation for $\mu\left(\omega_{\mathbf{q}}, \omega_{j}\right)$ and $z\left(\omega_{\mathbf{q}}\right)$ is obtained:

$$
\begin{aligned}
\mu\left(\omega_{\mathbf{q}}, \omega_{j}\right)\left(\omega_{\mathbf{q}}-\omega_{j}\right)= & \sum_{\mathbf{k} l} \frac{\mathbb{P}}{\omega_{\mathbf{q}}-\omega_{\mathbf{k}}} \mu\left(\omega_{\mathbf{q}}, \omega_{l}\right) W_{\mathbf{k} j} W_{\mathbf{k} l}^{*}+\sum_{\mathbf{k} l} \delta\left(\omega_{\mathbf{q}}\right. \\
& \left.-\omega_{\mathbf{k}}\right) z\left(\omega_{\mathbf{q}}\right) \mu\left(\omega_{\mathbf{q}}, \omega_{l}\right) W_{\mathbf{k} j} W_{\mathbf{k} l}^{*} .
\end{aligned}
$$

If we consider the nanoparticle to have a single mode, the relation between $\mu$ and $z$ can be written in a much simpler form and the dissipation of each mode can be treated separately. From this point on, we will use the subscript $j$ where necessary denoting that we are working on the dynamics of the $j$ th mode of the nanoparticle.

Relying on the above reasoning, $z_{j}\left(\omega_{\mathbf{q}}\right)$ can be expressed as

$$
z_{j}\left(\omega_{\mathbf{q}}\right)=\frac{\omega_{\mathbf{q}}-\omega_{j}-\sigma_{j}\left(\omega_{\mathbf{q}}\right)}{g\left(\omega_{\mathbf{q}}\right)\left|W_{\mathbf{q} j}\right|^{2}}
$$

$\sigma_{j}\left(\omega_{\mathbf{q}}\right)$ being the shift in the $j$ th nanoparticle mode, 


$$
\sigma_{j}\left(\omega_{\mathbf{q}}\right)=\mathbb{P} \int \frac{d \omega_{\mathbf{k}} g\left(\omega_{\mathbf{k}}\right)\left|W_{\mathbf{k} j}\right|^{2}}{\omega_{\mathbf{q}}-\omega_{\mathbf{k}}} .
$$

In order to obtain the expansion coefficients $\mu$ and $\nu$, phononic commutation relation for $\alpha\left(\omega_{\mathbf{q}}\right)$ is employed.

$$
\left[\alpha\left(\omega_{\mathbf{q}}\right), \alpha^{+}\left(\omega_{\mathbf{q}^{\prime}}\right)\right]=\delta_{\mathbf{q}, \mathbf{q}^{\prime}}=\frac{\delta\left(\omega_{\mathbf{q}}-\omega_{\mathbf{q}^{\prime}}\right)}{g\left(\omega_{\mathbf{q}}\right)} .
$$

Using the expansion in terms of bare operators [Eq. (B1)] and Poincare's theorem, i.e.,

$$
\begin{aligned}
\frac{P}{\omega_{\mathbf{q}}-\omega_{\mathbf{k}}} \frac{P}{\omega_{\mathbf{q}^{\prime}}-\omega_{\mathbf{k}}}= & \frac{\mathbb{P}}{\omega_{\mathbf{q}^{\prime}}-\omega_{\mathbf{q}}}\left(\frac{\mathbb{P}}{\omega_{\mathbf{q}}-\omega_{\mathbf{k}}}-\frac{P}{\omega_{\mathbf{q}^{\prime}}-\omega_{\mathbf{k}}}\right) \\
& +\pi^{2} \delta\left(\omega_{\mathbf{q}}-\omega_{\mathbf{k}}\right) \delta\left(\omega_{\mathbf{q}^{\prime}}-\omega_{\mathbf{k}}\right),
\end{aligned}
$$

the modulus square of $\mu\left(\omega_{\mathbf{q}}, \omega_{j}\right)$ is found as

$$
\left|\mu\left(\omega_{\mathbf{q}}, \omega_{j}\right)\right|^{2}=\frac{\left|W_{\mathbf{q} j}\right|^{2}}{\left[\omega_{\mathbf{q}}-\omega_{j}-\sigma_{j}\left(\omega_{\mathbf{q}}\right)\right]^{2}+\pi^{2} g^{2}\left(\omega_{\mathbf{q}}\right)\left|W_{\mathbf{q} j}\right|^{4}} .
$$

Since the Hamiltonian is diagonal with annihilation and creation operators $\alpha\left(\omega_{\mathbf{q}}\right)$ and $\alpha^{+}\left(\omega_{\mathbf{q}}\right)$ and eigenfrequencies $\omega_{\mathbf{q}}$, the time dependence of the dressed annihilation operator is

$$
\alpha\left(\omega_{\mathbf{q}}, t\right)=\mu\left(\omega_{\mathbf{q}}, \omega_{j}\right) a_{j} e^{-i \omega_{\mathbf{q}} t}+\sum_{\mathbf{k}} \nu\left(\omega_{\mathbf{q}}, \omega_{\mathbf{k}}\right) b_{\mathbf{k}} e^{-i \omega_{\mathbf{q}} t}
$$

Correspondingly, the time dependence of the nanoparticle annihilation operator reads [see Eq. (B2)]

$$
a_{j}(t)=\int d \omega_{\mathbf{q}} g\left(\omega_{\mathbf{q}}\right) \mu^{*}\left(\omega_{\mathbf{q}}, \omega_{j}\right) \alpha\left(\omega_{\mathbf{q}}\right) e^{-i \omega_{\mathbf{q}} t} .
$$

*On leave from Shadan Institute of P.G. Studies, Hyderabad 4, India.

†ciraci@fen.bilkent.edu.tr

${ }^{1}$ Physics of Sliding Friction, NATO Advanced Studies Institute, Series E: Applied Science, edited by B. N. J. Persson and E. Tosatti (Kluwer, Dordrecht, 1996), Vol. 311.

${ }^{2}$ Micro/Nanotribology and Its Applications, NATO Advanced Studies Institute, Series E: Applied Science, edited by B. Bhuhan (Kluwer, Dordrecht, 1997), Vol. 330.

${ }^{3}$ G. A. Tomlinson, Philos. Mag. 7, 905 (1929).

${ }^{4}$ J. Frenkel and T. Kontorova, Phys. Z. Sowjetunion 13, 1 (1938).

${ }^{5}$ B. Bhushan, J. N. Israelachvili, and U. Landman, Nature (London) 347, 607 (1995).

${ }^{6}$ A. P. Sutton and J. B. Pethica, J. Phys.: Condens. Matter 2, 5317 (1990).

${ }^{7}$ J. A. Nieminen, A. P. Sutton, and J. B. Pethica, Acta Metall. Mater. 40, 2503 (1992).

${ }^{8}$ M. R. Sorensen, K. W. Jacobsen, and P. Stoltze, Phys. Rev. B 53, 2101 (1996).

${ }^{9}$ M. R. Sorensen, K. W. Jacobsen, and H. Jonsson, Phys. Rev. Lett. 77, 5067 (1996)

${ }^{10}$ A. Buldum and S. Ciraci, Phys. Rev. B 55, 2606 (1997).

${ }^{11}$ A. Buldum and S. Ciraci, Phys. Rev. B 55, 12892 (1997).

${ }^{12}$ A. Buldum, S. Ciraci, and I. P. Batra, Phys. Rev. B 57, 2468 (1998).

${ }^{13}$ W. Zhong and D. Tomanek, Phys. Rev. Lett. 64, 3054 (1990).

${ }^{14}$ D. Tomanek, W. Zhong, and H. Thomas, Europhys. Lett. 15, 887 (1991).

${ }^{15}$ M. Cieplak, E. D. Smith, and M. O. Robins, Science 265, 1209 (1994).

${ }^{16}$ E. D. Smith, M. O. Robbins, and M. Cieplak, Phys. Rev. B 54,
8252 (1996).

${ }^{17}$ J. B. Sokoloff, Phys. Rev. B 42, 760 (1990).

${ }^{18}$ J. B. Sokoloff, Phys. Rev. B 42, 6745(E) (1990).

${ }^{19}$ J. B. Sokoloff, Phys. Rev. B 51, 15573 (1995).

${ }^{20}$ J. B. Sokoloff, Phys. Rev. Lett. 71, 3450 (1993).

${ }^{21}$ A. Buldum and S. Ciraci, Phys. Rev. B 60, 1982 (1999).

${ }^{22}$ A. Buldum, D. M. Leitner, and S. Ciraci, Phys. Rev. B 59, 16042 (1999).

${ }^{23}$ A. Buldum, S. Ciraci, and I. P. Batra, Phys. Rev. B 57, 2468 (1998).

${ }^{24}$ A. Erdemir, O. L. Eryilmaz, and G. Frenske, J. Vac. Sci. Technol. A 18, 1987 (2000).

${ }^{25}$ U. Fano, Phys. Rev. 124, 1866 (1961).

${ }^{26}$ P. W. Anderson, Phys. Rev. 124, 41 (1961).

${ }^{27}$ B. N. J. Persson and A. I. Volokitin, in Physics of Sliding Friction, NATO Advanced Studies Institute, Series E: Applied Science, edited by B. N. J. Persson and E. Tosatti (Kluwer, Dordrecht, 1996), Vol. 311, pp. 253-264.

${ }^{28}$ As long as $\left(\omega_{j} / \Gamma\right) \gg 1$ and $\left(\omega_{D} / \Gamma\right) \gg 1$ the Lorentzian is localized into the range where the Debye DOS is finite, so no extra contribution raises with extending the limits of integration.

${ }^{29}$ D. Vanderbilt, Phys. Rev. B 41, R7892 (1990).

${ }^{30}$ M. C. Payne, M. P. Teter, D. C. Allen, T. A. Arias, and J. D. Joannopoulos, Rev. Mod. Phys. 64, 1045 (1992).

${ }^{31}$ Numerical computations have been performed using VASP software. G. Kresse and J. Hafner, Phys. Rev. B 47, 558 (1993); G. Kresse and J. Furthmuller, ibid. 54, 011169 (1996).

${ }^{32}$ W. Kohn and L. J. Sham, Phys. Rev. 140, A1133 (1965); P. Hohenberg and W. Kohn, Phys. Rev. 136, B864 (1964).

${ }^{33}$ J. P. Perdew, K. Burke, and M. Ernzerhof, Phys. Rev. Lett. 77, 3865 (1996). 